\title{
Editorial
}

\section{Health impacts of chemical pesticides: The perspective of vegetable farmers}

Pesticides are an important tool used in intensive farming to maximize agricultural productivity and control pests, unwanted plants (weeds) and diseases. Farmers use pesticides in fruits, vegetables and cereals to control aphids, leaf rollers, caterpillars, worms, beetles, termites, and ants. Despite the advantages of pesticides, their intensive and widespread use can raise environmental and health consequences. In 1962, Rachel Carson documented the negative environmental effects caused by the injudicious use of pesticides through a book entitled "Silent Spring". In the developed world, pesticides used in agriculture are linked with many diseases. Recently, the Environmental Protection Agency (EPA), US demonstrated that the commonly used pesticide, chlorpyrifos. disrupt brain cells in children. Due to its toxicity, the Vietnamese government banned its use in early 2019. Likewise, triazophos has been banned in more than 40 countries. Besides agriculture, pesticides are used in public health to kill vectors of disease, such as mosquitoes in Nepal.

In the international journal, Science of the Total Environment, Bhandari and his colleagues recently published an article entitled Factors affecting pesticide safety behaviour: The perceptions of Nepalese farmers and retailers. They discovered that the most frequently self-reported toxicity symptoms related to pesticides were headache (73.8\%), skin irritation (62.3\%), eye irritation (32.8\%), weakness (22.4\%), and muscle pain (19.1\%). Likewise, other toxicity symptoms reported were shortness of breath, excessive sweating, vomiting, chapped hands, rhinorrhoea, throat discomfort, and salivation. The diseases are evident shorty after exposure or can arise within 48 hours of high exposure. Farmers in the study experienced the symptoms during spraying on their fields and sometimes symptoms lasted for 24 hours after the spray.

Chemical pesticides are responsible for many health issues, including carcinogens, mutagens, endocrine disrupters, reproductive effects, cholinesterase inhibitors, neurotoxins, respiratory tract irritants, skin and eye irritations and phototoxic development. In the journal, Environmental Research, Bhandari and his colleagues have recently published a second report entitled, Pesticide residues in Nepalese vegetables and potential health risks. Almost all (98\%) vegetable samples were contaminated with pesticide residues; up to 7 residues in a single sample. Of all the studied pesticides, they found residues of insecticides such as chlorpyrifos and triazophos in tomatoes at concentrations that cause health effects for consumers. 
According to Pesticide Action Network UK, continuous exposure to pesticide residues even at lower concentrations via consumption of foodstuffs, can cause chronic (long-term) toxicity in humans. Over time, they can cause serious diseases such as asthma, depression and anxiety, attention deficit, hyperactivity disorder, leukaemia, and non-Hodgkin's lymphoma.

Every year the number of patients suffering from cancer, cardiovascular diseases, the occurrence of renal failure, Alzheimer's disease, Parkinson's disease, and strokes are increasing in Nepal. Therefore, it is worth investigating whether or not these diseases are linked with the pesticides used in Nepal.

Nepal is a party of Basel, Rotterdam and Stockholm Conventions and should fulfil the obligations to minimise environmental pollution and to manage agro-chemicals, including pesticides. Pesticides such as profenofos, quinalphos, triazophos, and carbendazim are not approved for use in the EU due to their toxicity, persistence in the environment, likelihood of accumulation and bioamplification, impacting the long-term health of humans and the environment. These pesticides are still widely used in Nepal and should be banned immediately!

\section{Copyright $@ 2021 \mathrm{IJE}$}

This work is licensed under a CC BY-NC which permits use, distribution and reproduction in any medium provided the original work is properly cited and is not for commercial purposes

\section{Govinda Bhandari, PhD}

President, Progressive Sustainable Developers Nepal (PSD-Nepal) 